\title{
AVANCES Y RETOS EN LA CONSTRUCCIÓN DEL SISTEMA NACIONAL DE INVESTIGACIÓN EN SALUD EN EL PERÚ
}

\author{
Martín Yagui1,a, Manuel Espinoza ${ }^{1, b}$, Patricia Caballero' ${ }^{1, b}$, Teresa Castilla ${ }^{1, c}$, Gladys Garro ${ }^{1, c}$, \\ L. Patricia Yamaguchi ${ }^{1, \mathrm{c}}$, Henry Mormontoy ${ }^{1, \mathrm{~d}}$, Percy Mayta-Tristán ${ }^{2, \mathrm{~d}}$, Aníbal Velásquez $^{3, \mathrm{a}}$, \\ César Cabezas ${ }^{3, b}$
}

\begin{abstract}
RESUMEN
El objetivo de este artículo es presentar el estado del Sistema Nacional de Investigación en Salud de Perú (SNIS), las lecciones aprendidas en el proceso de construcción, las oportunidades de mejora y los retos. Se hace una descripción de las funciones del SNIS peruano, con relación a la gobernanza, el marco legal, la priorización de la investigación, el financiamiento, la creación y la sostenibilidad de recursos y la producción y utilización de la investigación. Se describe que en Perú se ejerce la gobernanza en la investigación, se cuenta con regulaciones, política y prioridades de investigación, estas últimas desarrolladas en el marco de un proceso participativo e inclusivo. Se concluye que los retos del SNIS peruano son consolidar la gobernanza y desarrollar los mecanismos para articular a los actores involucrados con la investigación, incrementar la asignación de recursos para la investigación e innovación, elaborar e implementar un plan para el desarrollo de recursos humanos dedicados a la investigación, desarrollar las instituciones y competencias regionales para hacer investigación, ligar la investigación para solucionar problemas y hacer sostenibles las políticas nacionales de investigación.
\end{abstract}

Palabras clave: Investigación científica y desarrollo tecnológico; Financiación de la investigación; Gestión de ciencia, tecnología e innovación en salud; Política de investigación en salud; Perú (fuente: DeCS BIREME).

\section{ADVANCES AND CHALLENGES IN BUILDING THE NATIONAL HEALTH RESEARCH SYSTEM IN PERU}

\begin{abstract}
The objective of this paper is to present the situational status of the National Health Research System of Peru (NHRS), the lessons learnt during the building process, the opportunities to improve it and the challenges. A description of the functions of the peruvian NHRS is done, in relation to governance, legal framework, research priorities, funding, creation and sustainability of resources and research production and utilization. It describes that in Peru we excert governance in research, we count with regulations, policy and research priorities, these last developed in the framework of a partipatory, inclusive process. The conclusion reached is that the challenges of the peruvian NHRS are to consolidate the governance and to develop the mechanisms to articulate the stakeholders involved in research, to improve the resources allocation for research and innovation, to ellaborate a plan for the development of human resources dedicated to research, to develop institutions and regional competences in order to perform research, and to link research in order to solve problems and make national research policies sustainable.
\end{abstract}

Key words: Scientific research and technological development; Research financing; Health sciences, techonology and innovation management; Health research policy; Peru (source: MeSH NLM).

\section{INTRODUCCIÓN}

El Perú se encuentra desarrollando reformas que están modificando el sistema de salud, particularmente se destaca el aseguramiento universal (1) y la descentralización de las funciones en materia de salud desde el nivel central al regional (2). Este nuevo escenario genera necesidades de información para la toma de decisiones y requiere la búsqueda de evidencias y la gestión del conocimiento para diseñar nuevas intervenciones, hacer más efectiva y eficientes las intervenciones y evaluar los cambios que se producen en la salud y bienestar.

\footnotetext{
1 Oficina General de Investigación y Transferencia Tecnológica, Instituto Nacional de Salud. Lima, Perú.

2 Oficina General de Información y Sistemas, Instituto Nacional de Salud. Lima, Perú.

3 Jefatura, Instituto Nacional de Salud. Lima, Perú.

a Médico epidemiólogo; ${ }^{b}$ Médico infectólogo; ' Enfermera epidemióloga; d Médico salubrista.
}

Recibido: 16-08-10 Aprobado: 08-09-10 
En respuesta a ello el Instituto Nacional de Salud (INS) ha conducido un proceso participativo para establecer las prioridades nacionales y regionales de investigación (3), las cuales cuentan con la legitimidad que le otorgan las instituciones públicas, privadas, académicas y de la sociedad civil que participaron. Estas prioridades son ahora la base para formular las políticas y para organizar el Sistema Nacional de Investigación en Salud (SNIS).

La investigación en salud es todo proceso dirigido a generar conocimiento sistemático y contrastar hipótesis para mejorar la salud y bienestar de la población. La investigación en salud, en el marco de los sistemas de salud, ha sido materia de muchas reuniones internacionales ${ }^{(4-6)}$, declaraciones, pronunciamientos e investigaciones sobre la materia. Probablemente uno de los más importantes lo constituye el "Reporte 10/90 sobre la investigación en salud" (7), en el cual estiman que los sectores públicos y privados gastan globalmente 56 mil millones de dólares anualmente en este tipo de investigación; sin embargo, menos del $10 \%$ de este monto se destina al $90 \%$ de los problemas de salud del mundo, situación calificada como el desequilibrio 10/90. También concluye que los países con rentas medias y bajas agrupan al $85 \%$ de la población mundial y soportan el $92 \%$ de la carga de enfermedad, mientras que los países ricos agrupan el $15 \%$ de la población mundial y subsecuentemente soportan el $8 \%$ de la carga de enfermedad ${ }^{(7)}$.

Algunas publicaciones sostienen que estos problemas se deben a la falta de gobernanza de la investigación en salud ${ }^{(8)}$, entendiéndose como gobernanza a Los procesos de acción colectiva que organizan la interacción entre los actores, la dinámica de los procesos y las reglas de juego (informales y formales), con las cuales una sociedad determina sus conductas, toma y ejecuta sus decisiones ${ }^{(9)}$, que incluye entre otros aspectos, las decisiones sobre qué tipo de investigaciones deben ser realizadas y financiadas con los recursos públicos. La gobernanza en la investigación en salud es básica para asegurar que la investigación sea éticamente conducida, tenga calidad científica y sea relevante para las poblaciones a las cuales va dirigida (10), por ello, la gobernanza es una de las cuatro funciones de los sistemas nacionales de salud.

La Organización Panamericana de la Salud (OPS), en su documento política de investigación en salud (11), define a los sistemas nacionales de investigación en salud como aquellos constituidos por los individuos e instituciones que rigen, administran, coordinan, requieren, crean, comunican o usan los datos resultantes de la investigación para promover, restablecer, mejorar o mantener el estado de salud y desarrollo de una población. Un SNIS nace de la intersección de dos grandes sistemas, el sistema de salud y el sistema de investigación, con la finalidad de generar y aplicar el conocimiento para mejorar la salud de las poblaciones ${ }^{(12)}$. Se reconoce cuatro funciones para dichos sistemas ${ }^{(11)}$ : 1 ) Gobernanza o rectoría, 2) Financiamiento, 3) Creación y sostenibilidad de recursos, 4) Producción y utilización de los resultados de la investigación.

En este contexto, el objetivo de esta publicación es presentar el estado del SNIS de Perú, las lecciones aprendidas en el proceso de construcción, las oportunidades de mejora y los retos.

\section{GOBERNANZA Y MARCO LEGAL}

\section{GOBERNANZA}

En general, la investigación, la ciencia y la tecnología en el Perú es articulada por el Consejo Nacional de Ciencia, Tecnología e Innovación Tecnológica (CONCYTEC), la cual es la institución rectora del Sistema Nacional de Ciencia y Tecnología e Innovación Tecnológica (SINACYT), integrada por la Academia, los institutos de investigación del Estado, las organizaciones empresariales, las comunidades y la sociedad civil ${ }^{(13)}$. Está regida por la Ley Marco de Ciencia y Tecnología $\mathrm{N} .{ }^{\circ}$ 28303. Tiene por finalidad normar, dirigir, orientar, fomentar, coordinar, supervisar y evaluar las acciones del Estado en el ámbito de la ciencia, tecnología e innovación tecnológica y promover e impulsar su desarrollo mediante la acción concertada y la complementariedad entre los programas y proyectos de las instituciones públicas, académicas, empresariales organizaciones sociales y personas integrantes del SINACYT. Tiene entre sus múltiples funciones la de promover la articulación de la investigación científica y tecnológica, y la producción del conocimiento con los diversos agentes económicos y sociales, para el mejoramiento de la calidad de vida y el impulso de la productividad y competitividad del país; implementar mecanismos de coordinación, intercambio y concertación entre las instituciones integrantes del SINACYT.

Por otro lado, el Ministerio de Salud (MINSA) a través del Instituto Nacional de Salud (INS) ejerce una función rectora en la investigación en salud y en la gestión de la investigación en salud en el Perú. Los mecanismos de coordinación ejercidos por diversas instancias del Estado, como por ejemplo el CONCYTEC, a través del SINACYT (promoviendo la investigación e integrando a los diversos actores públicos y privados de la investigación), por el MINSA a través del Consejo Nacional de Salud (mediante la participación activa de 
diversas instituciones del sector salud en su subcomité de investigación) y por el INS.

Durante este último decenio se impulsó la descentralización de varias funciones del Estado, una de las funciones de salud descentralizadas correspondió al desarrollo de recursos humanos en salud y dentro de esta función se descentralizó la función de gestión de la investigación (la descentralización de funciones se realizó hacia las regiones). Las facultades descentralizadas a las regiones con relación a la investigación son: a) Difundir y controlar la aplicación de normas nacionales de investigación en salud; b) Definir lineamientos regionales de investigación, en el marco de las políticas y normativa nacional; c) Identificar necesidades y prioridades regionales de investigación; d) Promocionar investigaciones en salud en la región, con participación intersectorial e interinstitucional y comunidad organizada; e) Programar, formular, aprobar $y$ ejecutar las investigaciones institucionales $y$; $f$ ) Difundir resultados de investigación en salud y realizar la transferencia tecnológica cuando corresponda.

Durante el año 2009, la Oficina de Descentralización del MINSA realizó un diagnóstico situacional de algunos indicadores en todas las funciones descentralizadas.
Para ello tomó como base los estándares e indicadores señalados en el documento denominado "Sistema de monitoreo y evaluación de la descentralización en salud" ${ }^{(14)}$. Los hallazgos en cuanto a la función de investigación a nivel regional mostraron que es el indicador con más bajo desempeño (Figura 1).

El año 2009, se evaluó la presencia y funcionamiento de las unidades de investigación de las 28 direcciones regionales de salud (DIRESA) del país, 43\% refirieron contar con una unidad de investigación pero solo una de ellas era parte de la estructura orgánica de su DIRESA, con escaso personal (uno en la mayoría de casos) que se dedica a varias funciones diferentes a la gestión de la investigación, situación que puede explicar el bajo nivel de avance en esta función transferida (Figura 1) ${ }^{(15)}$. Este bajo desempeño en cuanto a la función de investigación en las regiones puede obedecer a varias causas o factores, entre ellos la no adaptación de la organización de los gobiernos regionales y sus direcciones de salud a las nuevas funciones transferidas entre ellas la investigación, la insuficiente asignación de recursos financieros a la investigación a pesar que varios gobiernos regionales cuentan con recursos provenientes de regalías producto de la extracción

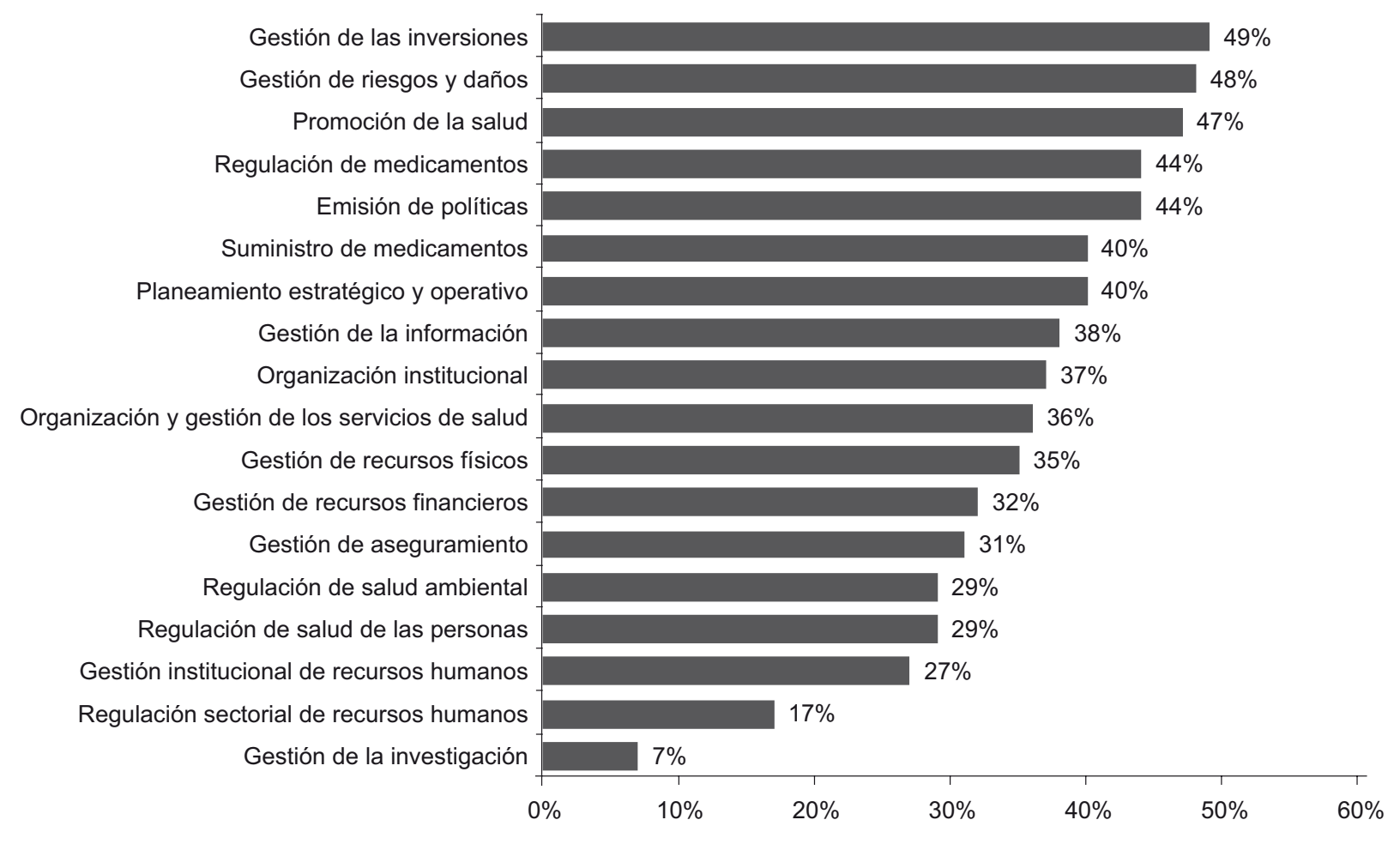

Figura 1. Avance promedio de las regiones en las funciones transferidas en el sector salud, Perú 2009. 
minera o petrolera y el poco tiempo de transferencia de estas nuevas funciones.

Los pasos que se vienen adoptando para el fortalecimiento de la gestión de la investigación a nivel nacional y regional son: a) Identificación de las prioridades regionales de investigación (3); b) Fortalecimiento de Unidades Regionales de Investigación en Salud, para ello se ha elaborado modelos de organización y funcionamiento de las mencionadas unidades para el nivel regional; c) Asistencia técnica para la realización de fondos concursables regionales para la investigación en salud, para ello se ha elaborado un modelo de bases de fondos concursables para el nivel regional; y d) Inicio del proceso de discusión de los lineamientos y políticas nacionales de investigación con las regiones.

\section{MARCO LEGAL}

El Perú cuenta con una estructura legal y regulatoria para la investigación en salud (Tabla 1) ${ }^{(16)}$, este marco legal se expresa desde la Constitución Política del Perú, en la cual en su artículo $14 .^{\circ}$ menciona que el Estado promueve el desarrollo científico y tecnológico. Por otro lado, la Ley Marco de Ciencia y Tecnología expresa en uno de sus artículos los principios fundamentales de la investigación y el rol del Estado en el desarrollo de la ciencia y tecnología. Paralelamente, en la Ley General de Salud se especifica el papel promotor del
Estado en la investigación científica y tecnológica en el campo de la salud y hace mención expresa de la investigación experimental con personas. Por otro lado, la Ley del Ministerio de Salud así como su reglamento, describen el papel rector del Instituto Nacional de Salud en la investigación en salud. En el año 2006 se emite el Decreto Supremo que Reglamenta los Ensayos Clínicos en Perú (17)

Si bien es cierto se cuenta con un marco legal vigente sobre investigación, existen retos pendientes de abordar. Un primer reto es la definición participativa y consensuada de la Política Nacional de Investigación, definiendo principios rectores, lineamientos generales y ejes programáticos. De igual forma es imperativo desarrollar dispositivos legales que permitan brindar incentivos para los investigadores nacionales. Actualmente se realiza una coordinación estrecha con el Consejo Nacional de Ciencia y Tecnología para el logro de estos retos, siendo el CONCYTEC parte del Consejo Consultivo del INS.

\section{PRIORIZACIÓN DE LA INVESTIGACIÓN}

En el Perú hay consenso en la necesidad de establecer prioridades nacionales de investigación, esto se refleja en las diversas experiencias de identificación de

Tabla1. Marco Legal que fundamenta la promoción y desarrollo de la investigación en salud por parte del Estado Peruano al año 2010.

\begin{tabular}{|c|c|}
\hline Leyes, Decretos y Reglamentos & Referencia específica a investigación \\
\hline Constitución Política del Perú & Art. N. ${ }^{\circ} 14$. Es deber del Estado promover el desarrollo científico y tecnológico del país \\
\hline $\begin{array}{l}\text { Ley Marco de Ciencia y Tecnología - } \\
\text { Ley N. }{ }^{\circ} 28303\end{array}$ & $\begin{array}{l}\text { Art. N. }{ }^{\circ} \text { 4. Principios fundamentales de las investigación... } \\
\text { Art. N. }{ }^{\circ} 5 \text {. Rol del Estado y los objetivos nacionales... }\end{array}$ \\
\hline Ley General de Salud - Ley N. ${ }^{\circ} 26842$ & $\begin{array}{l}\text { XV.- El Estado promueve la investigación científica y tecnológica en el campo de la } \\
\text { salud así como la formación, capacitación y entrenamiento de los recursos humanos } \\
\text { para el cuidado de la salud } \\
\text { Art. } \mathrm{N} \text {. }^{\circ} 15 \text { Toda persona, usuaria de los servicios de salud tiene derecho: a no ser } \\
\text { objeto de experimentación para la aplicación de medicamentos o tratamiento sin ser } \\
\text { debidamente informada sobre la condición experimental de estos, de los riesgos que } \\
\text { corre......... } \\
\text { Art. N. }{ }^{\circ} 28 \text { La investigación experimental con personas debe ceñirse a la legislación } \\
\text { especial sobre la materia y a los postulados éticos contenidos en la Declaración de } \\
\text { Helsinki y sucesivas declaraciones que actualicen los referidos postulados }\end{array}$ \\
\hline Ley del MINSA - Ley N. ${ }^{\circ} 27657$ & $\begin{array}{l}\text { Art.N. }{ }^{\circ} 33 \text { De las Misiones de los Organismos Públicos Descentralizados, a) El } \\
\text { Instituto Nacional de salud tiene como misión desarrollar y difundir la investigación y la } \\
\text { tecnología.... }\end{array}$ \\
\hline Reglamento de la Ley N. ${ }^{\circ} 27657$ & $\begin{array}{l}\text { Art. N. }{ }^{\circ} 34 \text { Instituto Nacional de Salud, es el organismo público descentralizado del } \\
\text { sector salud con autonomía técnica y de gestión, encargada de proponer políticas y } \\
\text { normas, promover, desarrollar y difundir la investigación científica-tecnológica.......... }\end{array}$ \\
\hline $\begin{array}{l}\text { Reglamento de Ensayos Clínicos del } \\
\text { Perú. Decreto Supremo N. } .^{\circ} 017-2006- \\
\text { SA, N. }{ }^{\circ} 006-2007-S A\end{array}$ & $\begin{array}{l}\text { Consta de } 12 \text { Títulos, } 137 \text { artículos ( } 33 \text { de los cuales fueron modificados en un segundo } \\
\text { decreto supremo) y disposiciones complementarias, transitorias y finales }\end{array}$ \\
\hline
\end{tabular}

Fuente: modificado de Cabezas ${ }^{(16)}$. 
Tabla 2. Prioridades nacionales de investigación en salud de Perú, 2009 - 2014 (3).

\begin{tabular}{lll}
\hline \multicolumn{1}{c}{ Área } & & \multicolumn{1}{c}{ Prioridad de Investigación } \\
\hline Recursos humanos en salud & 1. & Investigaciones para conocer los problemas de recursos humanos. \\
Salud mental & 2. & Investigaciones para conocer mejor la problemática de la salud mental. \\
Desnutrición infantil & 3. & Evaluación del impacto de los programas sociales estatales y no estatales sociales en la \\
& reducción de la desnutrición infantil.
\end{tabular}

prioridades que se han realizado desde 1974, a partir de dicho año se han producido hasta siete experiencias o iniciativas para la identificación de las prioridades de investigación en el país, cada una con características propias ${ }^{(18)}$.

La más reciente experiencia de priorización de la investigación en Perú se realizó en el segundo semestre del $2009^{(3)}$, este proceso de identificación de prioridades nacionales de investigación fue conducido por el INS, tuvo tres etapas: una etapa de consultas regionales, siendo su principal producto la identificación de las prioridades regionales de investigación; una segunda etapa de consulta de expertos, la cual congregó a los líderes de opinión y expertos temáticos en 14 áreas temáticas acordes con las prioridades sanitarias extraídas del Plan Nacional Concertado de Salud (19); y una tercera y última etapa de Foro Nacional, en donde en cuatro rondas deliberativas y de votación se eligieron, las prioridades sanitarias, los criterios de priorización, el número de prioridades y finalmente las prioridades de investigación.

Dicho proceso se implementó en seis meses aproximadamente, las características más importantes de esta experiencia fueron: fue un proceso participativo, inclusivo de todos los actores del sector salud y otros sectores relacionados con aspectos sanitarios, tanto públicos como privados, la academia y sociedades científicas, transparente (se contó con la supervisión de la Oficina Nacional de Procesos electorales de PerúONPE), descentralizado (se inició con la identificación de las prioridades regionales en casi todas las regiones del Perú y se culminó en un Foro Nacional), asociado con las prioridades sanitarias del país.

Las siete prioridades de investigación elegidas fueron áreas relacionadas con investigaciones sobre recursos humanos en salud, salud mental, mortalidad materna, desnutrición infantil y enfermedades transmisibles (Tabla
2). Mayores detalles de dicha experiencia se describen en la publicación de Caballero et al. (3). Es relevante mencionar que este proceso de priorización ha recibido el reconocimiento en julio de 2010 como una Buena Práctica de Gestión Gubernamental, reconocimiento promovido por la Organización no Gubernamental Ciudadanos al día, en el rubro de participación ciudadana.

Luego de la identificación de las prioridades de investigación, el INS viene impulsando el proceso de identificación de las agendas o líneas específicas de investigación para cada una de las prioridades. Para ello, se viene trabajando en procesos independientes para cada una de las áreas temáticas, en primer lugar se realizará una revisión de la evidencia o investigaciones realizadas en el país en los últimos diez años, seguidamente se identificará a todos los expertos, instituciones, grupos colaborativos y líderes de opinión que trabajan en el área priorizada y se realizaran entrevistas a profundidad con los líderes de opinión temáticos, en un tercer momento se realizarán encuentros que ayudaran a la discusión, deliberación, identificación y consenso de las líneas específicas de investigación.

Un reto inmediato es la implementación y ejecución de las investigaciones identificadas como prioritarias para el nivel nacional y regional.

\section{FINANCIAMIENTO}

El financiamiento de la investigación en el Perú tiene tres fuentes: pública, privada y cooperación internacional. El financiamiento de la investigación en salud en el sector público proviene principalmente del MINSA, a través del INS, institutos especializados de salud y del CONCYTEC. El financiamiento de la investigación del sector privado proviene de la industria farmacéutica y 
Tabla 3. Presupuesto anual del Instituto Nacional de Salud para investigaciones en salud según año, 2004-2009.

\begin{tabular}{cc}
\hline Año & Presupuesto aprobado en dólares \\
\hline 2004 & 2204236,28 \\
2005 & 2037490,96 \\
2006 & 2018969,11 \\
2007 & 2674026,85 \\
2008 & 2271658,53 \\
\hline 2009 & 2501736,90 \\
\hline
\end{tabular}

se concentra predominantemente en la ejecución de los ensayos clínicos multicéntricos autorizados en el país y en menor proporción por universidades privadas y organismos no gubernamentales. Por otro lado, la cooperación internacional tiene diversos patrocinadores en el país, así por ejemplo se han recibido fondos de las agencias cooperantes, del Fondo Mundial (centrado básicamente en tuberculosis, VIH/SIDA y malaria), de la Organización Panamericana de la Salud, universidades extranjeras y organismos públicos extranjeros como por ejemplo los Centros de Control de Enfermedades o los Institutos Nacionales de Salud de los EE.UU. Recientemente, se ha recibido financiamiento para investigaciones de la Red de Institutos Nacionales de Salud, del cual nuestro Instituto forma parte.

El Instituto Nacional de Salud, a través de su Oficina General de Investigación y Transferencia Tecnológica (OGITT) maneja un presupuesto anual promedio de dos millones de dólares, este presupuesto cubre aspectos de gestión de la investigación, ejecución de la investigación, gestión de la transferencia tecnológica, capacitación y entrenamiento del personal de salud (Tabla 3).
Según Garro et al. ${ }^{(20)}$ el presupuesto ejecutado por el INS para investigaciones en salud para el periodo 2004-2008 fue de 5918 640,43 dólares americanos, esto incluyó 182 proyectos de investigación aprobados y presupuestados, de los cuales se ejecutaron 150 (82,4\%), finalizaron 129 (86\%) y se publicaron 18 (13,9\%). De los 150 proyectos ejecutados, $60(40 \%)$ correspondió a investigaciones financiadas por un fondo concursable promovido por el INS en estos últimos 10 años. Posibles explicaciones del bajo porcentaje de investigaciones que culminaron en una publicación son: 1) desconocimiento por parte de los investigadores en cuanto a redacción de artículos científicos, 2) la falta de incentivos a los investigadores y 3) la dependencia del área de publicaciones a una oficina diferente a la Oficina General de Investigación y Transferencia Tecnológica, actualmente dicha área depende de la Oficina General de Sistemas e Información.

Es importante precisar que del total del presupuesto asignado en el período 2004 al 2008 (aproximadamente 11 millones de dólares) cerca de 5 millones fue ejecutado en el entrenamiento y capacitación del personal de salud del INS y de la red de laboratorios del MINSA en diversos temas relacionadas con las funciones asignadas, además de actividades propias de la gestión de la investigación y ejercicio de la regulación de la investigación experimental en seres humanos.

De igual forma, los institutos especializados de salud del país cuentan con oficinas o direcciones de investigación que realizan la gestión y promoción de la investigación, sus fuentes de financiamiento son diversas (Tabla 4).

Una de las principales dificultades que tiene el financiamiento público, es que no reconoce dentro de

Tabla 4. Fuentes de financiamiento para investigaciones según instituto especializado de salud, Ministerio de Salud.

\begin{tabular}{|c|c|c|c|c|c|}
\hline \multirow[b]{2}{*}{ Institutos } & \multirow[b]{2}{*}{ RDR } & \multirow{2}{*}{$\begin{array}{l}\text { Presupuesto } \\
\text { institucional }\end{array}$} & \multirow{2}{*}{$\begin{array}{c}\text { Financiado por } \\
\text { los propios } \\
\text { investigadores }\end{array}$} & \multicolumn{2}{|c|}{ Financiamiento externo } \\
\hline & & & & $\begin{array}{c}\text { Grants } \\
\text { internacionales }\end{array}$ & Otros \\
\hline $\begin{array}{l}\text { Instituto Nacional Especializado Materno } \\
\text { Perinatal }\end{array}$ & Sí & Sí & Sí & Sí & - \\
\hline Instituto Nacional de Salud del Niño & Sí & Sí & Sí & Sí & - \\
\hline Instituto Nacional de Salud Mental & Sí & Sí & Sí & - & - \\
\hline $\begin{array}{l}\text { Instituto Nacional de Enfermedades } \\
\text { Neoplásicas }\end{array}$ & Sí & - & Sí & Sí & Industria farmacéutica \\
\hline $\begin{array}{l}\text { Instituto Nacional de Ciencias } \\
\text { Neurológicas }\end{array}$ & - & - & - & Sí & - \\
\hline Instituto Nacional de Rehabilitación & - & Sí & - & - & $\begin{array}{l}\text { Oficina de Cooperación } \\
\text { Internacional }\end{array}$ \\
\hline Instituto Nacional de Oftalmología & Sí & Sí & - & - & - \\
\hline $\begin{array}{l}\text { Instituto Regional de Enfermedades } \\
\text { Neoplásicas del Sur }\end{array}$ & - & - & Sí & Sí & $\begin{array}{l}\text { Fondo Concursables } \\
\text { Nacionales }\end{array}$ \\
\hline
\end{tabular}

RDR: recursos directamente recaudados, se refiere a aquellos ingresos que obtuvo la institución diferente al presupuesto regular que le asigna el Estado. Presupuesto institucional: presupuesto regular que le asigna el Estado, también llamado Recursos Ordinarios (RO). Grants internacionales: Fondos financieros otorgado a investigadores nacionales o extranjeros provenientes de instituciones extranjeras 
los montos financiados el honorario de los investigadores por el tiempo dedicado a la investigación, por otro lado, este financiamiento no es administrado directamente por el investigador, sino que los fondos son transferidos a su institución de filiación, la cual solo puede ser del Estado, lo que genera dificultades para adquirir los insumos en el momento adecuado, entre otras cosas.

Así mismo, las normas que rigen este financiamiento, dificulta que investigadores independientes accedan a los financiamientos concursados por el Estado desde el sector salud. Se ha manejado opciones a través de consultorías para la ejecución de programas de investigación en temas específicos, como el de accidentes de tránsito ${ }^{(21)}$; sin embargo, aún no se ha evaluado el impacto de esta estrategia, por lo que se debe trabajar en este aspecto.

Un reto pendiente es el estudio y evaluación del financiamiento público para la investigación en salud, con la finalidad de contar con datos precisos que permitan realizar propuestas de mejora en cuanto al financiamiento, la calidad y su impacto en las políticas públicas en salud. La definición de las prioridades de investigación ayudará, en los próximos años, a direccionar el financiamiento público.

\section{CREACIÓN Y SOSTENIBILIDAD DE RECURSOS}

Existe en el Perú inequidad en la distribución del personal de salud, con una mayor concentración en Lima ${ }^{(22,23)}$ y un aumento en la emigración hacia países desarrollados, situación relacionada con una aspiración de mejora en sus ingresos económicos, entre otros factores ${ }^{(24)}$. Este escenario que se observa en el personal de salud en general, es más grave en aquellos profesionales capacitados o interesados en investigación, que suele ser escaso ${ }^{(25)}$ y, además, son los que emigran con mucho más frecuencia al encontrar un mayor campo e incentivos para su desarrollo (26-29), por lo que un fortalecimiento de los recursos humanos dedicados a investigación es necesario para el desarrollo de un SNIS ${ }^{(30)}$.

Un elemento básico para el desarrollo y sostenibilidad de los Sistemas Nacionales de Investigación es contar con profesionales dedicados a tiempo completo a la investigación, sin embargo, en el país no existe la carrera profesional de investigador, no hay puestos o posiciones presupuestadas como investigador en las entidades públicas de salud como por ejemplo en los Institutos especializados de salud en donde se comparte horas de trabajo asistencial con horas para investigación.
En Perú no existe un registro de los investigadores en salud ni en otras áreas de la ciencia, como se han venido desarrollando en otros países de la región, quienes a su vez, les dan ciertos incentivos según su nivel de productividad ${ }^{(31,32)}$, estas iniciativas son efectuadas por los Ministerios de Ciencia y Tecnología o los Consejos Superiores de Ciencia y Tecnología de cada país, aspecto que en el Perú, el trabajar en coordinación con el CONCYTEC, aún es un reto.

La formación de los investigadores está a cargo de las universidades; sin embargo, la mayoría de los programas de posgrado peruanos no son más que generadores de especialistas para el mercado laboral, que de recursos con habilidades para la investigación ${ }^{(33)}$. En ese sentido, a través de la Red de Institutos Nacionales de Salud de UNASUR ${ }^{(34)}$ y con el apoyo del Instituto Oswaldo Cruz de Brasil, se ha planificado la promoción de la formación de recursos humanos para los próximos años, tanto en salud pública como en otras áreas de la ciencia y tecnología.

Una alternativa para los investigadores jóvenes en formación, ha sido buscar oportunidades de capacitación fuera del país y trabajar en forma conjunta con investigadores reconocidos ${ }^{(35)}$, los cuales se encuentran agrupados en algunos centros de excelencia en la generación de conocimiento en Perú (36).

Se ha creado algunos mecanismos para evitar la fuga de talentos peruanos a países en desarrollo, como son las becas de retorno que algunas universidades vienen desarrollando desde hace algunos años ${ }^{(37)}$, el CONCYTEC ha creado recientemente un programa similar, y a partir del 2010 el INS ha accedido a la primera beca de reinserción de investigadores con grado de doctorado, la continuación de esta política de reinserción de recursos humanos altamente capacitados a los institutos del Estado es una oportunidad que debería ser expandida y potenciada.

Eldesarrollodelos recursoshumanosparalainvestigación en salud, incluye la formación de profesionales de diferentes partes del país en diversas áreas como por ejemplo bioestadística, metodología de la investigación, ética, redacción de artículos, buenas prácticas clínicas, entre otros, esto permitirá la implementación de las prioridades nacionales y regionales de investigación ${ }^{(3)}$. Adicionalmente, es conveniente identificar a aquellos estudiantes de pregrado que tienen un interés científico para que puedan ser orientados hacia una carrera en investigación, los cuales actualmente se encuentran agrupados en sociedades científicas de estudiantes de medicina ${ }^{(38,39)}$. 


\section{PRODUCCIÓN Y UTILIZACIÓN DE LA INVESTIGACIÓN}

El resultado de toda investigación debería terminar en una o más publicaciones, que permitan a los tomadores de decisiones usar el conocimiento para mejorar la salud de nuestra población ${ }^{(40,41)}$. Sin embargo, en nuestro país no es lo común que esto suceda, por ejemplo, de los proyectos de investigación financiados por el Instituto Nacional de Salud entre los años 2004 y 2008, solo $13,9 \%$ culminaban en publicación, mientras que las publicaciones de tesis de bachiller de la UPCH llegaron a17,6\% ${ }^{(42)}$, y las publicaciones de los trabajos de un curso de epidemiología para estudiantes de la facultad de medicina de la UNMSM fueron el $11,8 \%{ }^{(43)}$.

Parte de este problema, está relacionada con la poca capacitación en redacción de artículos ${ }^{(44)}$ y una ausencia de cultura de publicación con estímulos a ella ${ }^{(45) ;}$; situación que se ha venido revirtiendo a través de diferentes cursos realizados a nivel nacional, donde el Instituto Nacional de Salud, junto con los editores de revistas médicas peruanas, han tenido un papel destacado.

La investigación peruana en salud que logra ser publicada en revistas internacionales indizadas en ISI/Web of Knowledge, son escasas, pero están en incremento, así en el año 2000 se identificaron 121 artículos y 200 en el 2009. Esta producción está más relacionada con enfermedades infecciosas y tropicales y está concentrada en un grupo pequeño de instituciones, todas de Lima; adicionalmente, estas colaboran más intensamente con instituciones extranjeras que entre ellas ${ }^{(46)}$.

Sin embargo, a pesar de esta producción se tienen experiencias exitosas en el uso de los conocimientos producidos para el diseño de políticas de salud, ejemplo de ellos es el cambio de la política del tratamiento antimalárico, la cual tuvo como base estudios de la resistencia del Plasmodium falciparum; o la incorporación de la vacuna contra la hepatitis viral B en el Programa Ampliado de Inmunizaciones para niños menores de un año residentes en áreas de mediana y alta endemicidad, esta política sanitaria fue adoptada luego de la evidencia generada a partir de estudios de mortalidad en áreas endémicas y una posterior evaluación del impacto de la intervenciones (intervención piloto mediante la inmunización contra la hepatitis B en Abancay y luego en Huanta) ${ }^{(16)}$.

\section{DISCUSIÓN}

Hasta hace unos pocos años existía la idea generalizada de que la investigación era exclusividad de la academia y que el Estado a través de sus Ministerios debía mantenerse a cierta distancia de la investigación (47). Esto probablemente incrementó las inequidades y llevó a desequilibrios como los expresados en el reporte 10/90.

En Latinoamérica se han producido esfuerzos recientes por describir las características de los Sistemas Nacionales de investigación (SNIS) para la salud en por lo menos 14 países de la Región ${ }^{(48)}$. Este estudio descriptivo tuvo como fuente de información, informes preparados por expertos de los países que participaron en la Primera Conferencia Latinoamericana sobre Investigación e Innovación para la Salud (abril 2008, Río de Janeiro, Brasil) ${ }^{(5)}$. Se encontró que solo seis países cuentan con estructuras formales de gobernanza y gerencia de la investigación. Se concluyó que pese a la heterogeneidad estructural y funcional de los SNIS de los países analizados y su desigual nivel de progreso, se ha logrado avances en su desarrollo.

En el Perú, el Sistema Nacional de Investigación en Salud se está consolidando. Así por ejemplo, en la función de gobernanza se ha logrado un avance importante con la identificación de las Prioridades Nacionales de Investigación en salud durante el año 2009. Si bien es cierto hemos experimentado hasta siete momentos diferentes en la determinación de las prioridades nacionales de investigación en salud desde $1974{ }^{(18)}$, lo importante de este último proceso es la forma participativa y descentralizada, tomando en consideración las prioridades sanitarias expresadas en el Plan Nacional Concertado de Salud ${ }^{(3)}$. Estas prioridades han sido legitimadas por representantes de las regiones y del ámbito nacional y han sido promulgadas en una Resolución Ministerial el año 2010, por lo que tienen todas las condiciones para conducir el SNIS y orientar la asignación de recursos que provienen del Estado, la cooperación internacional y el sector privado.

Aún falta consolidar la gobernanza formalizando una Política Nacional de Investigación. Por ahora la política ha sido establecida de manera implícita cuando se establecieron las prioridades de investigación. Es decir, los criterios que se emplearon para establecer las prioridades ya definieron una política. En el proceso de priorización se determinó qué necesidades de investigación serían necesarias para hacer más efectivas y eficientes las intervenciones para atender las prioridades de salud del Plan Nacional Concertado de Salud. De esta forma la política nacional de investigación está ligada con las prioridades nacionales de salud y del sistema de salud.

Una debilidad en lagobernanza es lafalta de sostenibilidad de estas políticas dado que muchas veces dependen de las autoridades políticas de turno, en este sentido 
se debe buscar mecanismos que permitan asegurar que las políticas sean estables y puedan acompañarse de asignación de recursos y ser de amplia aceptación como para que puedan conducir el SNIS.

Una fortaleza con relación al marco legal en la investigación en salud en el último quinquenio fue la elaboración y posterior aprobación del Reglamento de Ensayos Clínicos (2006), el cual fue reconocido por varios referentes latinoamericanos como un instrumento valioso en la regulación de la investigación experimental con seres humanos; sin embargo 11 meses después de la mencionada aprobación, dicho reglamento sufrió la modificación de 33 artículos (2007) ${ }^{(17)}$, varios artículos modificados debilitaban la función reguladora del Estado, así por ejemplo, se eliminó el requisito de presentar copia del contrato entre el patrocinador, la organización de investigación por contrato, la institución de investigación e investigador principal (artículo 66), por otro lado, también en esta modificatoria se estableció excepciones para la presentación de pólizas de seguro (artículo 66), se suprimió la corresponsabilidad de la institución de investigación con el patrocinador y el investigador principal de los daños que sufra el sujeto de investigación consecuencia del ensayo clínico (artículo 55). Es preciso anotar que la modificación del reglamento generó mucha controversia. Actualmente se viene revisando el Reglamento de Ensayos Clínicos ante múltiples solicitudes de modificaciones en diversos aspectos, recogiendo las experiencias asimiladas desde el año 2006.

Una tarea pendiente, en el marco de la descentralización de las funciones del Estado, entre ellas salud, es la implementación o fortalecimiento según sea el caso de unidades $u$ oficinas regionales de investigación en salud, que permita a los gobiernos regionales la gestión de la investigación en sus ámbitos ${ }^{(15)}$.

En los últimos años se han producido diversas iniciativas para la formación de recursos humanos para la investigación en salud en el sector público, por ejemplo se han realizado entrenamientos en metodología de investigación, capacitaciones en ética en investigación, buenas prácticas clínicas, entre otras estrategias, sin embargo estas iniciativas no han estado enmarcadas en un plan nacional de capacitación que responda a las prioridades nacionales de investigación. Algunos sugieren que los investigadores deben formarse trabajando en proyectos relacionados con las prioridades de los sistemas nacionales de investigación en salud, dicha formación debe ser integral y articularse con el sector productivo ${ }^{(48)}$.

Con relación al financiamiento, son pocos los países que llegan a invertir el $1 \%$ del Producto Interno Bruto nacional en investigación en salud, siendo el promedio latinoamericano del 0,54, el Perú por ejemplo solo alcanza el $0,10 \%{ }^{(6)}$. Por otro lado, en recientes estudios ${ }^{(49)}$, se ha analizado el financiamiento público de la investigación en salud en cinco países de Latinoamérica (Argentina, Bolivia, Chile, Paraguay y Uruguay), encontraron que solo los países con mayores recursos o con redes de investigadores más desarrolladas poseen estructuras formales de asignación de fondos, con convocatorias periódicas y reglas estables.

El financiamiento público para la investigación en salud en Perú es aún reducido si observamos los presupuestos asignados históricamente en los últimos cinco años, esto guarda correlación con el gasto público en salud, el cual es uno de los más reducidos en Sudamérica (1,9\% como porcentaje del producto interno bruto para el año 2004). Por otro lado, una debilidad observada en el financiamiento de la investigación es que ante contextos de crisis económicas mundiales, desastres naturales $u$ otras emergencias sanitarias uno de los primeros presupuestos afectados en los recortes son los correspondientes a la investigación y formación de recursos humanos. Un aspecto pendiente es la realización de estudios sobre financiamiento de la investigación en salud en el sector público, no solamente para conocer con mayor aproximación los montos totales asignados, sino también su nivel de ejecución, su calidad de gasto y el impacto de las investigaciones financiadas y si estas realmente se han traducido en políticas de salud pública.

Analizando la producción científica de instituciones nacionales que si bien es cierto se ha incrementado en los últimos 10 años, aún es muy reducida si nos comparamos con otros países de Sudamérica ${ }^{(50)}$, aunque es importante tener en cuenta los niveles de desarrollo socioeconómico para una mejor valoración. Según Huamaní \& Mayta-Tristán (46), la producción científica nacional indizada en bases de datos internacionales se concentra en mayor porcentaje en unas pocas instituciones nacionales (más del $75 \%$ en cuatro instituciones nacionales). Se deben implementar mecanismos dentro del sector público para que más instituciones locales se involucren en investigación, así como también incrementar del porcentaje de proyectos de investigación financiados que culminan en publicaciones y que a su vez estas evidencias sirvan para la orientación o generación de las políticas públicas nacionales.

Una etapa necesaria de la investigación es la referida al intercambio de conocimiento mediante su publicación y la difusión eficaz de los resultados. En ese sentido, era necesario que exista una revista de salud pública en bases de datos internacionales, por ello es importante el 
logro del Instituto Nacional de Salud, al conseguir que la Revista Peruana de Medicina Experimental y Salud Pública esté indizada en Medline, constituyéndose en una ventana al mundo para mostrar las investigaciones que se realizan en el país y la región ${ }^{(51)}$.

Según la publicación realizada por Alger et al. ${ }^{(48)}$ no existe aún el Sistema Nacional de Investigación en Salud en el Perú, porque no existe gobernanza para la investigación ni política dedicada e inclusiva; sin embargo con el presente artículo se puede sostener que sí existe gobernanza, existen regulaciones, política y prioridades de investigación. Lo que hace falta es consolidar la gobernanza y desarrollar los mecanismos para articular a los actores involucrados con la investigación, incrementar la asignación de recursos para la investigación e innovación, hacer un plan para el desarrollo de recursos humanos dedicados a la investigación, desarrollar las instituciones y competencias regionales para hacer investigación, ligar la investigación para solucionar problemas, atender la carga de enfermedad actual, mejorar la eficacia y eficiencia de las intervenciones sanitarias y hacer sostenibles las políticas nacionales de investigación.

Como se observa se tiene fortalezas y debilidades en el proceso de construcción del Sistema Nacional de Investigación en Salud en Perú. Finalmente es importante reafirmar lo mencionado en la Primera Conferencia Latinoamericana sobre investigación e innovación para la salud ${ }^{(6,52)}$ : la rectoría de los Sistemas Nacionales de investigación en Salud, incluyendo la investigación, el desarrollo y la distribución de tecnología, es responsabilidad indelegable del Estado.

\section{AGRADECIMIENTOS}

Al personal de Instituto Nacional de Salud que con su trabajo vienen contribuyendo a mejorar el estado de salud de la población peruana.

\section{Conflictos de Interés}

Los autores trabajan en las diferentes áreas del Instituto Nacional de Salud involucradas en la construcción del SNIS del Perú.

\section{REFERENCIAS BIBLIOGRÁFICAS}

1. Ugarte O. Aseguramiento universal en salud en el Perú. Rev Peru Med Exp Salu Publica. 2009; 26(2): 133.

2. Perú, Ministerio de Salud. Descentralización de la función salud al nivel local. Los proyectos piloto. Lima: MINSA; 2007.
3. Caballero P, Yagui M, Espinoza M, Castilla T, Granados A, Velásquez A, et al. Prioridades regionales y nacionales de investigación en salud, Perú 2010-2014: un proceso con enfoque participativo y descentralista. Rev Peru Med Exp Salud Publica. 2010;27(3):367-72.

4. Cumbre Ministerial sobre Investigación en Salud. Declaración de México sobre las investigaciones sanitarias. Conocimientos para una mejor salud: fortalecimiento de los sistemas de salud. México DF: OMS; 2004.

5. Consejo de Investigación en Salud para el Desarrollo (COHRED), Organización Panamericana de la Salud. $1^{\text {a }}$ Conferencia Latinoamericana sobre Investigación e Innovación para la Salud. Informe de la conferencia. Rio de Janeiro: COHRED, OPS; 2008.

6. Consejo de Investigación en Salud para el Desarrollo (COHRED), Organización Panamericana de la Salud. Reunión de Seguimiento a la $1^{\text {a }}$ Conferencia Latinoamericana sobre investigación e innovación para la salud: informe final. La Habana: COHRED, OPS; 2009

7. Global Forum for Health Research. The 10/90 report on health research 2000. Geneva: Global Forum for Health Research; 2000.

8. Lee K, Mills A. Strengthening governance for global health research. BMJ. 2000; 321: 775-76.

9. Huffy M, Báscolo E, Bazzani R. Gobernanza en salud: un aporte conceptual y analítico para la investigación. Cad Saude Publica. 2006; 22(Suppl): S35-45

10. De los Ríos R. Gobernanza de la investigación función esencial de la salud pública. Elementos para la discusión. Washington DC: OPS; 2005.

11. Organización Panamericana de la Salud. Política de investigación para la salud. Washington DC: OPS; 2009.

12. Pang T, Sadana R, Hanney S, Bhutta ZA, Hyder AA, Simon J. Knowledge for better health-a conceptual framework and foundation for health research systems. Bull World Health Organ. 2003; 81(11): 815-20.

13. Consejo Nacional de Ciencia y Tecnología. Plan nacional de ciencia, tecnología e innovación tencológica para el desarrollo productivo y social sostenible 2008-2012. Lima: CONCYTEC; 2008.

14. Perú, Ministerio de Salud. Sistema de monitoreo y evaluación de la descentralización en salud. Ejercicio de las Funciones Descentralizadas en salud en el ámbito regional, 2009. Lima: MINSA; 2009.

15. Yamaguchi LP, Yagui M, Mayta-Tristán P. Las unidades regionales de investigación en salud, Perú 2009. Rev Peru Med Exp Salud Publica. 2010;27(3):480-81.

16. Cabezas $\mathbf{C}$. Rol del Estado en la investigación científica en salud y transparencia en la información. Rev Peru Med Exp Salud Publica. 2006; 23(4): 275-83.

17. Instituto Nacional de Salud (INS). Reglamento de ensayos clínicos. Lima: INS; 2010.

18. Instituto Nacional de Salud. Prioridades de investigación en salud en el Perú: análisis del proceso. Lima: INS; 2007.

19. Perú, Ministerio de Salud (MINSA). Plan nacional concertado de salud. Lima: MINSA; 2007.

20. Garro G, Mormontoy H, Yagui M. Gestión y financiamiento de las investigaciones por el Instituto Nacional de Salud, Perú 2004-2008. Rev Peru Med Exp Salud Publica. 2010;27(3):361-66. 
21. Miranda JJ, Huicho L. Traumatismos causados por el tránsito en el Perú. ¿Dónde estamos y hacia dónde vamos? Rev Peru Med Exp Salud Publica. 2010; 27(2): 157-61.

22. Arroyo J. Los sistemas descentrados de recursos humanos en salud: el caso del Perú, 1990-2005. Cienc Saude Coletiva. 2006;11(4):1063-72.

23. Carrasco V, Lozano E, Velásquez E. Análisis actual y prospectivo de la oferta y demanda de médicos en el Perú 2005-2011. Acta Med Peru. 2008;25(1):22-29.

24. Mayta-Tristán P, Dulanto-Pizzorni D, Miranda JJ. Low wages and brain drain: an alert from Peru. Lancet. 2008;371:1577.

25. Salas SP, Rigotti A. Médicos científicos en Chile: ¿una especie en extinción? Rev Med Chile. 2005;133(1):121-28.

26. Kaushik M, Roy A, Bang AA, Mahal A. Quality of medical training and emigration of physicians from India. BMC Health Serv Res. 2008;8:e279.

27. Tugwell P, Sitthi-Amorn C, Hatcher-Roberts J, Neufeld V, Makara P, Munoz F, et al. Health research profile to assess the capacity of low and middle income countries for equityoriented research. BMC Public Health. 2006;6:e151.

28. Pitayarangsarit $S$, Tangcharoensathien V. Sustaining capacity in health policy and systems research in Thailand. Bull World Health Organ. 2009;87(1):72-74.

29. Fiestas F, Gallo C, Poletti G, Bustamante I, Alarcón RD, Mari JJ, et al. What challenges does mental and neurological health research face in Latin American countries. Rev Bras Psiquiatr. 2008;30(4):328-36.

30. Lansang MA, Dennis R. Building capacity in health research in the developing world. Bull World Health Organ. 2004; 82(10):764-70.

31. Marcano D, Phélan M. Evolución y desarrollo del programa de promoción del investigador en Venezuela. Interciencia. 2009; 34(1): 17-24.

32. Colombo S, Bergonzelli P. Balance de 10 años de políticas públicas de ciencia y tecnología en Argentina. Aportes. 2006;11(33):57-84.

33. Murillo JP, Mendoza W, Franco G. ¿Quo vadis salud pública? Discuso y práctica de la salud pública en el Perú y su auge universitario de los noventa. An Fac Med (Lima). 2000;61(2):146-60.

34. Velásquez A, Cabezas C. Nuevos desafíos para los Institutos Nacionales de Salud de América del Sur: la globalización, las reformas sanitarias y la cooperación sursur. Rev Peru Med Exp Salud Publica 2009;26(4):419-21.

35. Garcia PJ, Curioso WH. Strategies for aspiring biomedical researchers in resource-limited environments. PLoS Negl Trop Dis. 2008;2(8):e274.

36. Cáceres CF, Mendoza W. Globalized research and "national science" the case of Peru. Am J Public Health. 2009; 99(10): 1792-98.

37. Guerra H. La beca de retorno de la Universidad Peruana Cayetano Heredia. Rev Peru Med Exp Salud Publica. 2010;27(3):326-36.

38. Mayta-Tristán P. Sociedades científicas de estudiantes de medicina: el futuro de la investigación en Latinoamérica. MedUNAB. 2010;13(1):3-4.

39. Villafuerte-Galvez J, Curioso WH, Miranda JJ. The role of medical students in the fight to control neglected tropical diseases: a view from Peru. PLoS Negl Trop Dis. 2008;2(9):e292.

40. Kilama W. From research to control: translating research findings into health policies, operational guidelines and health products. Acta Trop. 2009; 112(Suppl 1): S91-101.

41. Fiestas F. La investigación como herramienta para mejorar el área de la salud mental y neurológica en países con pocos recursos. Rev Neuropsiquiatr. 2009;72(1-4):47-57.

42. Arriola-Quiroz I, Curioso WH, Cruz-Encarnación M, Gayoso O. Characteristics and publication patterns of theses from a Peruvian medical school. Health Info Libr J. 2010;27(2):148-54.

43. Alarcón-Villaverde J, Romaní f, Gutiérrez C. Publicaciones científicas estudiantiles producidas en el curso de epidemiología de la Facultad de Medicina de la Universidad Nacional Mayor de San Marcos durante el periodo 20032009. An Fac Med (Lima). 2010;71(2):111-16.

44. Molina-Ordoñez J, Huamaní C, Mayta-Tristán $P$. Apreciación estudiantil sobre la capacitación universitaria en investigación: estudio preliminar. Rev Peru Med Exp Salud Publica. 2008;25(3):325-29.

45. Gutierrez C, Mayta P. Publicación desde el pre grado en Latinoamérica: importancia, limitaciones y alternativas de solución. CIMEL. 2003;8(1):54-60.

46. Huamani C, Mayta-Tristán P. Producción científica peruana en medicina y redes de colaboración, análisis del Science Citation Index 2000-2009. Rev Peru Med Exp Salud Publica. 2010;27(3):315-25.

47. Cáceres CF, Cuba V. Investigación científica y políticas públicas en salud: memoria de las I jornadas científicas en salud pública. Lima: Universidad Peruana Cayetano Heredia; 2007.

48. Alger J, Becerra-Posada F, Kennedy A, Martinelli E, Cuervo LG, Grupo Colaborativo de la Primera Conferencia Latinoamericana de investigación e Innovación para la Salud. Sistemas nacionales de investigación para la salud en América Latina: una revisión de 14 países. Rev Panam Salud Publica. 2009; 26(5): 447-57.

49. Maceira D, Paraje G, Aramayo F, Duarte Masi S, Sánchez D. Financiamiento público de la investigación en salud en cinco países de América Latina. Rev Panam Salud Publica. 2010; 27(6): 442-51.

50. Razzouk D, Gallo C, Olifson S, Zorzetto R, Fiestas F, Polleti G, et al. Challenges to reduce the '10/90 gap': mental health research in Latin American and Caribbean countries. Acta Psychiatr Scand. 2008;118(6):490-98.

51. Burstein Z, Mayta-Tristán P. La Revista Peruana de Medicina Experimental y Salud Pública ahora indizada en MEDLINE/Index Medicus. Rev Peru Med Exp Salud Publica. 2010;27(3):312-14.

52. Becerra-Posada F. Nuevas posibilidades de desarrollo de los sistemas nacionales de investigación para la salud en América Latina. Salud Publica Mex. 2009; 51(1): 86-89.

Correspondencia: Dr. Martín Yagui Moscoso

Dirección: Cápac Yupanqui 1400, Lima 11, Perú.

Teléfono: (511) 617-6200 anexo: 2144

Correo electrónico: myagui@ins.gob.pe 